\title{
Quasilinear Degenerated Elliptic Systems with Weighted in Divergence Form with Weak Monotonicity with General Data
}

\author{
Abdelkrim Barbara, El Houcine Rami, Elhoussine Azroul \\ Laboratory LAMA, Department of Mathematics, Faculty of Sciences Dhar El Mahraz, Sidi Mohammed Ben Abdellah University, \\ Atlas Fez, Morocco \\ Email: babdelkarim66@hotmail.com,ramielhoucine@gmail.com, elhoussine.azroul@gmail.com
}

How to cite this paper: Barbara, A., Rami, E.H. and Azroul, E. (2021) Quasilinear Degenerated Elliptic Systems with Weighted in Divergence Form with Weak Monotonicity with General Data. Applied Mathematics, 12, 500-519.

https://doi.org/10.4236/am.2021.126035

Received: October 11, 2020

Accepted: June 27, 2021

Published: June 30, 2021

Copyright $\odot 2021$ by author(s) and Scientific Research Publishing Inc. This work is licensed under the Creative Commons Attribution International License (CC BY 4.0).

http://creativecommons.org/licenses/by/4.0/

\section{Abstract}

We consider, for a bounded open domain $\Omega$ in $\mathbb{R}^{n}$ and a function $u: \Omega \rightarrow \mathbb{R}^{m}$, the quasilinear elliptic system:

$(Q E S)_{(f, g)} \begin{cases}-\operatorname{div} \sigma(x, u(x), D u(x))=v(x)+f(x, u, D u)+\operatorname{divg}(x, u) & \text { in } \Omega \\ u=0 & \text { on } \partial \Omega,\end{cases}$

(1). We generalize the system $(Q E S)_{(f, g)}$ in considering a right hand side depending on the jacobian matrix $D u$. Here, the star in $(Q E S)_{(f, g)}$ indicates that $f$ may depend on $\mathrm{Du}$. In the right hand side, $v$ belongs to the dual space $W^{-1, p^{\prime}}\left(\Omega, \omega^{*}, R^{m}\right),\left(\frac{1}{p}+\frac{1}{p^{\prime}}=1, p>1\right), f$ and $g$ satisfy some standard continuity and growth conditions. We prove existence of a regularity, growth and coercivity conditions for $\sigma$, but with only very mild monotonicity assumptions.

\section{Keywords}

Quasilinear Elliptic, Sobolev Spaces with Weight, Young Measure, Galerkin Scheme

\section{Introduction}

In this paper, the main point is that we do not require monotonicity in the strict monotonicity of a typical Leray-Lions operator as it is usually assumed in previous papers. The aims of this text are to prove analogous existence results under relaxed monotonicity, in particular under strict quasi-monotonicity. The main 
technical tool we advocate and use throughout the proof is Young measures. By applying a Galerkin schema, we obtain easily an approximating sequence $u_{k}$. The Ball theorem [1] and especially the resulting tool mode available by Hungerbühler to partial differential equation theory give them a sufficient control on the gradient approximating sequence $D u_{k}$ to pass to the limit. This method is used by Dolzmann [2], G. J. Minty [3], H. Brezis [4], H. E. Stromberg [5], Muller [6], J. L. Lions [7], Kristznsen, J. Lower [8], M. I. Visik [9] and mainly by Hungurbühler to get the existence of a weak solution for the quasi-linear elliptic system [10]. This paper can be seen as generalization of Hungurbühler and as a continuation of Y-Akdim [11].

This kind of problems finds its applications in the model of Thomas-Fermis in atomic physics [12], and also porous flow modeling in reservoir [13].

\section{Priliminaries}

Let $\omega=\left\{\omega_{i j} ; 0 \leq i \leq n ; 1 \leq j \leq m\right\}$ the weight function systems defined in $\Omega$ and satisfying the following integrability conditions:

$$
\begin{aligned}
& \left.\omega_{i j} \in L_{l o c}^{1}(\Omega), \omega_{i j}^{\frac{-1}{p-1}} \in L_{l o c}^{1}(\Omega), \text { for some } p \in\right] 1, \infty[ \\
& \text { and } \exists s>0 \text { such that } \omega_{i j}^{-s} \in L^{1}(\Omega) .
\end{aligned}
$$

with $\omega^{*}=\left\{\omega_{i j}^{*}=\omega_{i j}^{1-p^{\prime}}, 0 \leq i \leq n, 1 \leq j \leq m\right\}, \quad \sigma=\left(\sigma_{r s}\right)$ with $1 \leq s \leq n, 1 \leq r \leq m$ and which satisfies some hypotheses (see below).

We denote by $I M^{m \times n}$ the real vector space of $m \times n$ matrices equipped with the inner product $M: N=\sum_{i j} M_{i j} N_{i j}$.

The Jacobian matrix of a function $u: \Omega \rightarrow \mathbb{R}^{m}$ is denoted by $D u(x)=\left(D_{1} u(x), D_{2} u(x), \cdots, D_{n} u(x)\right)$ with $D_{i}=\partial / \partial\left(x_{i}\right)$.

The space $W^{1, p}\left(\Omega, \omega, \mathbb{R}^{m}\right)$ is the set of functions

$$
\begin{aligned}
& \left\{u=u(x) / u \in L^{p}\left(\Omega, \overline{\omega_{0}}, \mathbb{R}^{m}\right)\right\}, D_{i j} u=\frac{\partial u^{i}}{\partial x_{j}} \in L^{p}\left(\Omega, \omega_{i j}, \mathbb{R}^{m}\right), \\
& 1 \leq i \leq n, 1 \leq j \leq m .
\end{aligned}
$$

with

$$
L^{p}\left(\Omega, \omega_{i j}, \mathbb{R}^{m}\right)=\left\{u=u(x) /|u| \omega_{i j}^{\frac{1}{p}} \in L^{p}\left(\Omega, \mathbb{R}^{m}\right)\right\}
$$

The weighted space $W^{1, p}\left(\Omega, \omega, \mathbb{R}^{m}\right)$ can be equipped by the norm:

$$
\|u\|_{1, p, \omega}=\left(\sum_{j=1}^{m} \int_{\Omega}\left|u_{j}\right|^{p} \omega_{0 j} \mathrm{~d} x+\sum_{1 \leq i \leq n, 1 \leq j \leq m} \int_{\Omega}\left|D_{i j} u\right|^{p} \omega_{i j} \mathrm{~d} x\right)^{\frac{1}{p}}
$$

where $\overline{\omega_{0}}=\left(\omega_{0 j}\right)$ and $1 \leq j \leq m$. the norm $\|\cdot\|_{1, \omega, p}$ is equivalent to the norm $\|\mid\| \cdot \|$, on $W_{0}^{1, p}\left(\Omega, \omega, \mathbb{R}^{m}\right)$, such that, \|\|$u \| \mid=\left(\sum_{1 \leq i \leq n, 1 \leq j \leq m} \int_{\Omega}\left|D_{i j} u\right|^{p} \omega_{i j} \mathrm{~d} x\right)^{\frac{1}{p}}$.

Proposition 2.1 The weighted Sobolev space $W^{1, p}\left(\Omega, \omega, \mathbb{R}^{m}\right)$ is a Banach space, separable and reflexive. The weighted Sobolev space $W_{0}^{1, p}\left(\Omega, \omega, \mathbb{R}^{m}\right)$ is the 
closure of $C_{0}^{\infty}\left(\Omega, \omega, \mathbb{R}^{m}\right)$ in $W^{1, p}\left(\Omega, \omega, \mathbb{R}^{m}\right)$ equipped by the norm $\|\cdot\|_{1, p, \omega}$.

Proof: The prove of proposition is a slight modification of the analogous one in [14] [Kufner-Drabek].

Definition 2.1 A Young measure $\left(\vartheta_{x}\right)_{x \in \Omega}$ is called $W^{1, p}$-gradient young measures $(1 \leq p<\infty)$ if it is associated to a sequence of gradients $D u_{k}$ such that $u_{k}$ is bounded in $W^{1, p}(\Omega)$. The $W^{1, p}$-gradient young measures $\left(\vartheta_{x}\right)_{x \in \Omega}$ is called homogeneous, if it doesn't depend on $x, i$-e, if $\vartheta_{x}=\vartheta$ for a.e. $x \in \Omega$.

Theorem 2.1 (Kinder, Lehirer-Pedregal) let $\left(v_{x}\right)_{x \in \Omega}$, be a family of probability measures in $\left(C\left(M^{m \times n}\right)\right)$. Then, $\left(v_{x}\right)_{x \in \Omega}$ is $W^{1, p}$ Young measures if and only if:

1) There is a $u \in W^{1, p}\left(\Omega, \mathbb{R}^{m}\right)$ such that $D u(x)=\int_{M^{m \times n}} \operatorname{Ad}_{\vartheta_{x}}(A)$, a.e in $\Omega$.

2) Jensen's inequality: $\phi(D u(x)) \leq \int_{M^{m \times n}} \phi(A) \mathrm{d} \vartheta_{x}(A)$ hold for all $\phi \in X^{p}$ quasi-convex, and.

3) The function: $\psi(x)=\int_{M^{m \times n}}|A|^{p} \mathrm{~d} \vartheta_{x}(A) \in L^{1}(\Omega)$. Here, $X^{p}$ denotes the (not separable) space:

$X^{p}=\left\{\psi \in C\left(M^{m \times n}\right):|\psi(A)| \leq c \times\left(1+|A|^{p}\right)\right.$, for all $\left.A \in M^{m \times n}\right\}$.

proof: see [15].

Theorem 2.2 (Ball) Let $\Omega \in \mathbb{R}^{n}$ be Lebesgue measurable, let $K \in \mathbb{R}^{m}$ be closed, and let $u_{j}: \Omega \rightarrow \mathbb{R}^{m}, j \in \mathbb{N}$, be a sequence of Lebesgue measurable functions satisfying $u_{j} \rightarrow K$, as $j \rightarrow \infty$, i.e. given any open neighborhood $U$ of $K \in \mathbb{R}^{m}, \lim _{j \rightarrow \infty}\left|x \in \Omega: u_{j}(x) \in U\right|=0$. Then there exists a subsequence $u_{k}$ of $u_{j}$ and a family $\vartheta_{x}, x \in \Omega$, of positive measures on $I^{m}$, depending measurably on $x$, such that

1) $\left\|\vartheta_{x}\right\|_{M}=\int_{\mathbb{R}^{m}} \mathrm{~d} \vartheta_{x} \leq 1$, for a.e $x \in \Omega$.

2) $\operatorname{supp} \vartheta_{x} \subset K$ for a.e $x \in \Omega$.

3) $f\left(u_{k}\right) \rightarrow^{*}\left\langle\vartheta_{x}, f\right\rangle=\int_{\mathbb{R}^{m}} f(\lambda) \mathrm{d} \vartheta_{x}(\lambda)$ in $L^{\infty}(\Omega)$. for each continuous functions $f: \mathbb{R}^{m} \rightarrow \mathbb{R}$ satisfying

$\lim f(\lambda)=0,|\lambda| \rightarrow \infty \quad[1]$.

Theorem 2.3 (vitali) Let $\Omega \in \mathbb{R}^{n}$ be an open bounded domain and let $u_{n}$ be a sequence in $L^{p}\left(\Omega, \mathbb{R}^{m}\right)$ with $1 \leq p<\infty$.

Then $u_{n}$ is a cauchy sequence in the $L^{p}$-norm if and only if the two following conditions hold:

1) $u_{n}$ is cauchy in measure (i.e.: $\forall \varepsilon>0,\left|\left\{x \in \Omega,\left|u_{n}(x)-u_{m}(x)\right| \geq \varepsilon\right\}\right|=0$ as $m, n \rightarrow \infty$.

2) $\left|u_{n}\right|^{p}$ is equiintegrable i.e.:

$\operatorname{(sup}_{n} \int_{\Omega}\left|u_{n}\right|^{p} \mathrm{~d} x<\infty$ and $\forall \varepsilon>0, \exists \delta>0$ such that $\int_{E}\left|u_{n}\right|^{p} \mathrm{~d} x<\varepsilon$ for all $n$ whenever $E \subset \Omega$ and $|E|<\delta$ ). Note that if $u_{n}$ converges pointiest, then $u_{n}$ is cauchy in measure.

Hypotheses $\left(H_{0}\right)$ (Hardy-Type inequalities): There exist some constant $c>0$, some weighted function $\gamma$ and some real $q(1<q<\infty)$ such that,

$$
\left(\sum_{j=1}^{m} \int_{\Omega}\left|u_{j}(x)\right|^{q} \gamma_{j}(x) \mathrm{d} x\right)^{\frac{1}{q}} \leq c\left(\sum_{1 \leq i \leq n, 1 \leq j \leq m} \int_{\Omega}\left|D_{i j} u\right|^{p} \omega_{i j}\right)^{\frac{1}{p}},
$$


for all $u \in W_{0}^{1, p}\left(\Omega, \omega, \mathbb{R}^{m}\right)$, with $\gamma=\left\{\gamma_{j} / 1 \leq j \leq m\right\}$.

The injection $W_{0}^{1, p}\left(\Omega, \omega, \mathbb{R}^{m}\right) \hookrightarrow \hookrightarrow L^{q}\left(\Omega, \gamma, \mathbb{R}^{m}\right)$ is compact, and $W_{0}^{1, p}\left(\Omega, \omega, \mathbb{R}^{m}\right) \hookrightarrow \hookrightarrow L^{r}\left(\Omega, \mathbb{R}^{m}\right)$ is compact, (by [14]) with

$$
\begin{cases}1 \leq r \prec \frac{n p s}{n(s+1)-p s} & \text { if } p s \prec n(s+1) \\ r \geq 1 & \text { if } n(s+1) \prec p s\end{cases}
$$

$\left(H_{1}\right)$ Continuity: $\sigma: \Omega \times \mathbb{R}^{m} \times I M^{m \times n} \rightarrow I M^{m \times n}$ is a Carathéodory function (i-e $x \mapsto \sigma(x, u, F)$ is measurable for every $(u, F) \in \mathbb{R}^{m} \times \mathbb{M}^{m \times n}$ and $(u, F) \mapsto \sigma(x, u, F)$ is continuous for almost every $x \in \Omega)$. ( $\left.H_{2}\right)$ Growths and coercivity conditions: There exist $c_{1} \geq 0, c_{2}>0, \lambda_{1} \in L^{p^{\prime}}(\Omega), \lambda_{2} \in L^{1}(\Omega)$, $\lambda_{3} \in L^{(p / \alpha)^{\prime}}(\Omega), \quad 0<\alpha<p, \quad 1<q<\infty$ and $\beta>0$ such that for all $1 \leq r \leq n, 1 \leq s \leq m$, we have:

$$
\left|\sigma_{r s}(x, u, F)\right| \leq \beta w_{r s}^{1 / p}\left[\lambda_{1}(x)+c_{1} \sum_{j=1}^{m}\left|\gamma_{j}\right|^{1 / p^{\prime}} \cdot\left|u_{j}\right|^{q / p^{\prime}}+c_{1} \sum_{1 \leq i \leq N, 1 \leq j \leq m} \omega_{i j}^{1 / p^{\prime}}\left|F_{i j}\right|^{p-1}\right]
$$

and

$$
\sigma(x, u, F): F \geq-\lambda_{2}(x)-\sum_{j=1}^{m} \omega_{0 j}(x)^{\alpha / p} \lambda_{3}(x)\left|u_{j}\right|^{\alpha}+c_{2} \sum_{1 \leq i \leq n, 1 \leq j \leq m} \omega_{i j}(x) \cdot\left|F_{i j}\right|^{p}
$$

$\left(H_{3}\right)$ Monotonicity conditions: $\sigma$ satisfies one of the following conditions:

1) For all $x \in \Omega$, and all $u \in \mathbb{R}^{m}$, the map $F \mapsto \sigma(x, u, F)$ is a $C^{1}$-function and is monotone (i-e, $(\sigma(x, u, F)-\sigma(x, u, G)):(F-G) \geq 0$, for all $x \in \Omega$, all $u \in \mathbb{R}^{m}$ and all $\left.F, G \in I M^{m \times n}\right)$.

2) There exists a function $W: \Omega \times \mathbb{R}^{m} \times I M^{m \times n} \rightarrow I M^{m \times n}$ such that $\sigma(x, u, F)=\frac{\partial W}{\partial F}(x, u, F)$ and $F \mapsto W(x, u, F)$ is convex and $C^{1}$ function.

3) For all $x \in \Omega$, and for all $u \in \mathbb{R}^{m}$ the map $F \mapsto \sigma(x, u, F)$ is strictly monotone (i.e., $\sigma(x, u,$.$) is monotone and:$ $[(\sigma(x, u, F)-\sigma(x, u, G)):(F-G)=0] \Rightarrow F=G)$.

4) $\sigma(x, u, F)$ is strictly p-quasi-monotone in $F$, i.e.,

$$
\int_{M^{m \times n}}(\sigma(x, u, \lambda)-\sigma(x, u, \bar{\lambda})):(\lambda-\bar{\lambda}) \mathrm{d} \vartheta(\lambda)>0,
$$

for all homogeneous $W^{1, p, w}$-gradient young measures $\vartheta$ with center of mass $\bar{\lambda}=\langle\vartheta$, id $\rangle$ which are not a single Dirac mass.

The main point is that we do not require strict monotonicity or monotonicity in the variables $(u, F)$ in $\left(H_{3}\right)$ as it is usually assumed in previous work (see [15] or [16]).

$\left(F_{0}\right)^{*}:$ (continuity) $f: \Omega \times \mathbb{R}^{m} \times I M^{m \times n} \rightarrow \mathbb{R}^{m}$ is a Carathéodory function i-e: $x \mapsto f(x, u, F)$ is measurable for every $u \in \mathbb{R}^{m}$, and $F \in M^{m \times n}$, $(u, F) \mapsto f(x, u, F)$ is continuous for almost every $x \in \Omega$.

$\left(F_{1}\right)^{*}:$ (growth condition): The exist: $b_{1} \in L^{p^{\prime}}(\Omega), \quad c_{1}^{\prime}>0, \quad c_{2}^{\prime}>0$ such that:

$$
\left|f_{j}(x, u)\right| \leq\left[b_{1}(x)+c_{1}^{\prime} \gamma_{j}^{\frac{1}{p^{\prime}}}\left|u_{j}\right|^{\frac{q}{p^{\prime}}}+c_{2}^{\prime} \sum_{r, s} \omega_{r s}^{\frac{1}{P^{\prime}}}\left|F_{r s}\right|^{p-1}\right] \omega_{0 j}^{\frac{1}{P}} ;
$$


$\forall 1 \leq j \leq m \quad\left(G_{0}\right)^{*}:$ (continuity) the map $g: \Omega \times \mathbb{R}^{m} \rightarrow I M^{m \times n}$ is a Carathéodory function. $\left(G_{1}\right)^{*}:$ (growth condition) There exist: $b_{2} \in L^{p^{\prime}}(\Omega)$

$$
\left|g_{r s}\right| \leq \omega_{r s}^{\frac{1}{p}}\left[b_{2}+\sum_{j} \gamma_{j}^{\frac{1}{p^{\prime}}}\left|u_{j}\right|^{\frac{q}{p^{\prime}}}\right]
$$

For all $1 \leq r \leq n$ and $1 \leq s \leq m$.

Our aim of this paper is to prove the existence of the problem $(Q E S)_{f, g}$ in the space $W_{0}^{1, P}\left(\Omega, \omega, R^{m}\right)$.

Remark 2.1 -The condition $\left(F_{0}\right)^{*}$ and $\left(G_{0}\right)$ ensure the measurability of $f$ and $g$ for all measurable function $u$.

- $\left(F_{1}\right)$ and $\left(G_{1}\right)^{*}$ ensure that growths conditions, in particularly: if $u \in W_{0}^{1, P}\left(\Omega, \omega, \mathbb{R}^{m}\right)$ then $f(., u(),. D().) \cdot u($.$) and g(., u): D u$ is in $L^{1}(\Omega, \omega)$.

- Exploiting the convergence in measure of the gradients of the approximating solutions, we will prove the following theorem.

Theorem 2.4 If $p \in(1, \infty)$ and $\sigma$ satisfies the conditions $\left(H_{0}\right)-\left(H_{3}\right)$, then the Dirichlet problem $(Q E S)_{f, g}^{*}$ has a weak solution $u \in W_{0}^{1, p}\left(\Omega, \omega, \mathbb{R}^{m}\right)$, for every $v \in W^{-1, p^{\prime}}\left(\Omega, \omega^{*}, \mathbb{R}^{m}\right)$, f satisfies $\left(F_{0}\right)^{*}$ and $\left(F_{1}\right)^{*}$ and $g$ satisfies $\left(G_{0}\right)$ and $\left(G_{1}\right)$.

In order to prove theorems, we will apply a Galerkin scheme, with this aim in view, we establish in the following subsections, the key ingredient to pass to the limit for this, we assume that the conditions: $\left(H_{0}\right)-\left(H_{3}\right),\left(F_{0}\right)^{*},\left(F_{1}\right)^{*},\left(G_{0}\right)$ and $\left(G_{1}\right)$.

Lemma 2.1 For arbitrary $u \in W_{0}^{1, p}\left(\Omega, \omega, \mathbb{R}^{m}\right)$ and $v \in W^{-1, p^{\prime}}\left(\Omega, \omega^{*}, \mathbb{R}^{m}\right)$, the functional

$$
\begin{aligned}
& F(u): W_{0}^{1, p}\left(\Omega, \omega, \mathbb{R}^{m}\right) \rightarrow \mathbb{R} \\
& \varphi \mapsto \int_{\Omega} \sigma(x, u(x), D u(x)): D \varphi(x) \mathrm{d} x-\langle v, \varphi\rangle \\
& \quad-\int_{\Omega} f(x, u, D u): \varphi \mathrm{d} x+\int_{\Omega} g(x, u): D \varphi \mathrm{d} x .
\end{aligned}
$$

is well defined, linear and bounded.

Proof For all $\varphi \in W_{0}^{1, p}\left(\Omega, \omega, \mathbb{R}^{m}\right)$, we denote

$$
F(u)(\varphi)=I_{1}+I_{2}+I_{3}+I_{4}
$$

with

$$
I_{1}=\int_{\Omega} \sigma(x, u(x), D u(x)): D \varphi(x) \mathrm{d} x,
$$

and

$$
\begin{gathered}
I_{2}=-\langle v, \varphi\rangle . \\
I_{3}=-\int_{\Omega} f(x, u, D u): \varphi \mathrm{d} x \\
I_{4}=\int_{\Omega} g(x, u): D \varphi \mathrm{d} x
\end{gathered}
$$

We define

$$
I_{r s}=\int_{\Omega} \sigma_{r s}(x, u(x), D u(x)): D_{r s} \varphi(x) \mathrm{d} x
$$


Firstly, by virtue of the growth conditions $\left(H_{2}\right)$ and the Hölder inequality, one has

$$
\begin{aligned}
\left|I_{r s}\right| \leq & \int_{\Omega}\left|\sigma_{r s}(x, u(x), D u(x))\right|:\left|D_{r s} \varphi(x)\right| \mathrm{d} x \\
\leq & \int_{\Omega} \beta \omega_{r s}^{1 / p}(x)\left[\lambda_{1}(x)+c_{1} \sum_{j=1}^{m}\left|\gamma_{j}(x)\right|^{1 / p^{\prime}}\left|u_{j}(x)\right|^{q / p^{\prime}}\right. \\
& +c_{1} \sum_{1 \leq i \leq n, 1 \leq j \leq m} \omega_{i j}^{1 / p^{\prime}}\left|D_{i j} u\right|^{p-1}|| D_{r s} \varphi \mid \mathrm{d} x \\
\leq & \beta\left[\left(\int_{\Omega}\left|\lambda_{1}(x)\right|^{p^{\prime}} \mathrm{d} x\right)^{1 / p^{\prime}}\left(\int_{\Omega}\left|D_{r s} \varphi(x)\right|^{p} \omega_{r s} \mathrm{~d} x\right)^{1 / p}\right. \\
& +\left(\int_{\Omega}\left|D_{r s} \varphi(x)\right|^{p} \omega_{r s}\right)^{1 / p}\left(\sum_{j=1}^{m} \int_{\Omega}\left|u_{j}\right|^{q} \gamma_{j} \mathrm{~d} x\right)^{1 / p^{\prime}} \\
& \left.+\left(\sum_{1 \leq i \leq n, 1 \leq j \leq m} \int_{\Omega}\left|D_{i j} u\right|^{p} \omega_{i j} \mathrm{~d} x\right)^{1 / p^{\prime}}\left(\int_{\Omega}\left|D_{r s} \varphi\right|^{p} \omega_{r s} \mathrm{~d} x\right)^{1 / p}\right]
\end{aligned}
$$

with $\left(p=p^{\prime}(p-1)\right)$, and thanks to Hardy inequality we have:

$$
\begin{aligned}
\left|I_{r s}\right| & \leq c \beta\left[\left\|\lambda_{1}\right\|_{p^{\prime}}\|\varphi\|_{1, p, \omega_{r s}}+c_{1}\|D \varphi\|_{p, \omega_{r s}}\left(\int_{\Omega}|u|^{q} \gamma \mathrm{d} x\right)^{1 / p^{\prime}}+c_{1} \sum_{i j}\|D \varphi\|_{p, \omega_{i j}}\|D u\|_{p, \omega_{r s}}\right] \\
& \leq c^{\prime} \beta\left[\left\|\lambda_{1}\right\|_{p^{\prime}}\|\varphi\|_{1, p, \omega_{r s}}+\|\varphi\|_{1, p, \omega_{r s}}\|u\|_{q, \gamma}+\|u\|_{1, p, \omega}\|\varphi\|_{1, p, \omega_{r s}}\right]
\end{aligned}
$$

with $c^{\prime}=\max (c, 1)$. Which gives

$$
\left|I_{1}\right| \leq c^{\prime} \beta\left[\left\|\lambda_{1}\right\|_{p^{\prime}}+\|u\|_{1, p, \omega}^{q / p^{\prime}}+\|u\|_{1, p, \omega}\right]\|\varphi\|_{1, p, \omega}<\infty .
$$

and

$$
\begin{gathered}
\left|I_{2}\right| \leq \int_{\Omega}\left|v\|\varphi \mid \mathrm{d} x \leq\| v\left\|_{-1, p^{\prime}, \omega^{*}}\right\| \varphi \|_{1, p, \omega}<\infty .\right. \\
I_{3}=\sum_{j} \int_{\Omega} f_{j}(x, u, D u) \varphi_{j}(x) \mathrm{d} x
\end{gathered}
$$

We denote $I_{3, j}=\left|\int_{\Omega} f_{j}(x, u, D u) \varphi_{j}(x) \mathrm{d} x\right|$.

$$
\begin{aligned}
I_{3, j} \leq & \int_{\Omega}\left|f_{j}(x, u, D u)\right|\left|\varphi_{j}(x)\right| \mathrm{d} x \\
\leq & \int_{\Omega} b_{1}(x)\left|\varphi_{j}(x)\right| \omega_{0 j}^{\frac{1}{p}} \mathrm{~d} x+c_{1}^{\prime} \int_{\Omega} \gamma_{j}^{\frac{1}{p^{\prime}}}\left|u_{j}\right|^{\frac{q}{p^{\prime}}}\left|\varphi_{j}(x)\right| \omega_{0 j}^{\frac{1}{p}} \\
& +c_{2}^{\prime} \int_{\Omega} \sum_{r s}\left|\varphi_{j}(x)\right|\left|D_{r s} u\right|^{p-1} \omega_{0 j}^{\frac{1}{p}} \mathrm{~d} x \\
\leq & \left(\int_{\Omega}\left|b_{1}(x)\right|^{p^{\prime}}\right)^{\frac{1}{p^{\prime}}}\left(\int_{\Omega}\left|\varphi_{j}(x)\right|^{p} \omega_{0 j} \mathrm{~d} x\right)^{\frac{1}{p}} \\
& +\left(\int_{\Omega} \gamma_{j}(x)\left|u_{j}\right|^{q} \mathrm{~d} x\right)^{\frac{1}{p^{\prime}}}\left(\int_{\Omega}\left|\varphi_{j}(x)\right|^{p} \omega_{0 j} \mathrm{~d} x\right)^{\frac{1}{p}} \\
& +\sum_{r s}\left(\int_{\Omega}\left|\varphi_{j}\right|^{p} \omega_{0 j}\right)^{\frac{1}{p}} \cdot\left(\int_{\Omega}\left|D_{r s} u\right|^{p^{\prime}(p-1)} \omega_{r s}\right)^{\frac{1}{p^{\prime}}} \\
\leq & \left\|b_{1}\right\|_{p^{\prime}}\|\varphi\|_{1, p, \omega}+c_{1}^{\prime}\left(\sum_{j} \int_{\Omega} \gamma_{j}(x)\left|u_{j}\right|^{q} \mathrm{~d} x\right)^{\frac{1}{p^{\prime}}} \cdot\|\varphi\|_{1, p, \omega}+c_{2}^{\prime}\|\varphi\|_{1, \omega, p} \cdot\|D u\|_{1, p, \omega}^{\frac{p}{p^{\prime}}}
\end{aligned}
$$




$$
\begin{gathered}
\leq\left\|b_{1}\right\|_{p^{\prime}}\|\varphi\|_{1, p, \omega}+c_{1}^{\prime} \cdot\|D u\|_{1, p, \omega} \cdot\|\varphi\|_{1, p, \omega}+c_{2}^{\prime}\|\varphi\|_{1, \omega, p} \cdot\|D u\|_{1, p, \omega}^{\frac{p}{p^{\prime}}} \\
\leq\left(\left\|b_{1}\right\|+c_{1}^{\prime} \cdot\|D u\|_{1, p, \omega}+c_{2}^{\prime} \cdot\|D u\|_{1, p, \omega}^{\frac{p}{p^{\prime}}}\right) \cdot\|\varphi\|_{1, p, \omega} \cdot \\
I_{4}=\sum_{r s} \int_{\Omega} g_{r s}(x, u) D_{r s} \varphi \mathrm{d} x \\
\int_{\Omega}\left|g_{r s}\right|:\left|D_{r s} \varphi\right| \mathrm{d} x \\
\leq \int_{\Omega} b_{2} \omega_{r s}^{\frac{1}{p}} \cdot D_{r s} \varphi \mathrm{d} x+\sum_{j} \int_{\Omega} \gamma_{j}^{\frac{1}{p^{\prime}}}(x)\left|u_{j}\right|^{\frac{q}{p^{\prime}}} \omega_{r s}^{\frac{1}{p}} D_{r s} \varphi \mathrm{d} x \\
\leq\left(\int_{\Omega}\left|b_{2}\right|^{p^{\prime}} \mathrm{d} x\right)^{\frac{1}{p^{\prime}}} \cdot\left(\int_{\Omega}\left|D_{r s} \varphi\right|^{p} \omega_{r s} \mathrm{~d} x\right)^{\frac{1}{p}} \\
+\sum_{j}\left(\int_{\Omega}\left|u_{j}\right|^{q} \gamma_{j}(x) \mathrm{d} x\right)^{\frac{1}{p^{\prime}}}\left(\int_{\Omega}\left|D_{r s} \varphi\right|^{p} \omega_{r s}(x) \mathrm{d} x\right)^{\frac{1}{p}} \\
\leq\left\|b_{2}\right\|_{p^{\prime}}\left\|D_{r s} \varphi\right\|_{1, p, \omega_{r s}}+\|u\|_{q, \gamma}^{\frac{q}{p^{\prime}}}\left(\int_{\Omega}\left|D_{r s} \varphi\right|^{p} \omega_{r s} \mathrm{~d} x\right)^{\frac{1}{p}} \\
I_{4} \leq\left\|b_{2}\right\|_{p^{\prime}}\left\|D_{r s} \varphi\right\|_{1, p, \omega_{r s}}+\|u\|_{q, \gamma}^{\frac{q}{p^{\prime}}}\left(\int_{\Omega}\left|D_{r s} \varphi\right|^{p} \omega_{r s} \mathrm{~d} x\right)^{\frac{1}{p}} \\
\leq\left\|b_{2}\right\|_{p^{\prime}} \cdot\|D \varphi\|_{1, p, \omega}+\|u\|_{q, \gamma}^{\frac{q}{p^{\prime}}} \cdot\|D \varphi\|_{1, p, \omega} \\
\leq c^{\prime \prime}\|\varphi\|_{1, p, \omega}
\end{gathered}
$$

Hence $I \leq c_{4}\|\varphi\|_{1, p, \omega}$. With $c_{4}<\infty$.

Finally the functional $F($.$) is bounded.$

Lemma 2.2 The restriction of $F$ to a finite dimensional linear subspace $V$ of $W_{0}^{1, p}\left(\Omega, \omega, \mathbb{R}^{m}\right)$ is continuous.

Proof Let $d$ be the dimension of $V$ and $\left(e_{1}, e_{2}, \cdots, e_{d}\right)$ a basis of $V$. Let $u_{j}=\sum a_{j}^{i} \cdot e_{i}$ be a sequence in $V$ which converges to $u=\sum a^{i} e_{i}$ in $V$. The sequence $\left(a_{j}\right)$ converge to $a \in \mathbb{R}^{d}$, so $u_{j} \rightarrow u$ and $D u_{j}^{1<i \leq d} \rightarrow D u$ a.e., on the other hand $\left\|u_{j}\right\|_{p}$ and $\left\|D u_{j}\right\|_{p}$ are bounded by a constant $c$. Thus, it follows by the continuity conditions $\left(H_{1}\right)$, that

$$
\sigma\left(x, u_{j}, D u_{j}\right): D \varphi \rightarrow \sigma(x, u, D u): D \varphi
$$

for all $\varphi \in W_{0}^{1, p}\left(\Omega, \omega, \mathbb{R}^{m}\right)$ and a.e. in $\Omega$. Let $\Omega^{\prime}$ be a measurable subset of $\Omega$ and let $\varphi \in W_{0}^{1, p}\left(\Omega, \omega, \mathbb{R}^{m}\right)$.

Thanks to the condition $\left(H_{2}\right)$, we get

$$
\int_{\Omega^{\prime}}\left|\sigma\left(x, u_{j}, D u_{j}\right): D \varphi\right| \mathrm{d} x<\infty,
$$

By the continuity conditions $\left(F_{0}\right)^{*}$ and $\left(G_{0}\right)$ we have:

$$
f\left(x, u_{j}, D u_{j}\right) \cdot \varphi \rightarrow f(x, u, D u) \cdot \varphi
$$

And

$$
g\left(x, u_{j}\right) \cdot D \varphi \rightarrow g(x, u) \cdot D \varphi
$$


almost everywhere. Moreover we infer from the growth conditions $\left(F_{1}\right)^{*}$ and $\left(G_{1}\right)$ that the sequences:

$$
\left(\sigma\left(x, u_{j}, D u_{j}\right): D \varphi\right),\left(f\left(x, u_{j}, D u_{j}\right) \cdot \varphi\right) \text { and }\left(g\left(x, u_{j}\right) \cdot D \varphi\right)
$$

Are equi-integrable. Indeed, if $\Omega^{\prime} \subset \Omega$ is a measurable subset and $\varphi \in W_{0}^{1, p}\left(\Omega, \omega, \mathbb{R}^{m}\right)$ then:

$\int_{\Omega^{\prime}}\left|f\left(x, u_{j}, D u_{j}\right) \cdot \varphi\right| \mathrm{d} x<\infty \quad$ (by $\left(F_{1}\right)^{*}$ and Hölder inequality).

$\int_{\Omega^{\prime}} g\left(x, u_{j}\right) \cdot D \varphi \mid \mathrm{d} x<\infty \quad$ (by $\left(G_{1}\right)$ and Hölder inequality).

$\int_{\Omega^{\prime}}\left|\sigma\left(x, u_{j}, D u_{j}\right): D \varphi\right| \mathrm{d} x<\infty$ (by Hölder inequality).

which implies that $\sigma\left(x, u_{j}, D u_{j}\right): D \varphi$ is equi-integrable. And by applying the Vitali's theorem, it follows that

$$
\int_{\Omega} \sigma\left(x, u_{j}, D u_{j}\right): D \varphi \mathrm{d} x \rightarrow \int_{\Omega} \sigma(x, u, D u): D \varphi \mathrm{d} x,
$$

for all $\varphi \in W_{0}^{1, p}\left(\Omega, \omega, \mathbb{R}^{m}\right)$.

Finally

$$
\lim _{j \rightarrow \infty}\left\langle F\left(u_{j}\right), \varphi\right\rangle=\langle F(u), \varphi\rangle,
$$

which means that

$$
F\left(u_{j}\right) \rightarrow F(u) \text { in } W^{-1, p^{\prime}}\left(\Omega, \omega^{*}, \mathbb{R}^{m}\right) .
$$

Remark 2.2 Now, the problem $(Q E S)_{f, g}^{*}$ is equivalent to find a solution $u \in W_{0}^{1, p}\left(\Omega, \omega, \mathbb{R}^{m}\right)$ such that $\langle F(u), \varphi\rangle=0$, for all $\varphi \in W_{0}^{1, p}\left(\Omega, \omega, \mathbb{R}^{m}\right)$.

In order to find such a solution we apply a Galerkin scheme.

\section{Galerkin Approximation}

\section{Remark 3.1 (Galerkin Schema)}

Let $V_{1} \subset V_{2} \subset \cdots \subset W_{0}^{1, p}\left(\Omega, \omega, \mathbb{R}^{m}\right)$ be a sequence of finite dimensional subspaces with $\bigcup_{k \in \mathbb{N}} V_{k}$ dense in $W_{0}^{1, p}\left(\Omega, \omega, \mathbb{R}^{m}\right)$. The sequence $V_{k}$ exists since $W_{0}^{1, p}\left(\Omega, \omega, \mathbb{R}^{m}\right)$ is separable.

Let us fix some $k$, we assume that $V_{k}$ has a dimension $d$ and that $\left(e_{1}, e_{2}, \cdots, e_{d}\right)$ is a basis of $V_{k}$. Then, we define the map,

$$
\begin{aligned}
& G: \mathbb{R}^{k} \rightarrow \mathbb{R}^{k} \\
& \quad\left(a_{1}, \cdots, a_{k}\right) \mapsto\left(\left\langle F(u), e_{1}\right\rangle, \cdots,\left\langle F(u), e_{k}\right\rangle\right) ; u=\sum_{i=1}^{d} a_{i} e_{i} .
\end{aligned}
$$

Proposition 3.1 The map $G$ is continuous and $G(a) \cdot a$ tends to infinity when $\|a\|_{\mathbb{R}^{k}}$ tends to infinity.

Proof. Since $F$ restricted to $V_{k}$ is continuous by Lemma 2.2, so $G$ is continuous.

Let $a \in \mathbb{R}^{d}$ and $u=\sum a^{i} \cdot e_{i}$ in $V_{k}$, then $G(a) \cdot a=\langle F(u), u\rangle$ and which implies that $\|a\|_{\mathbb{R}^{d}}$ teicd tends to infinity if $\|u\|_{1, p, \omega}$ tends to infinity.

$$
G(a) \cdot a=\sum_{1 \leq i \leq d}\left\langle F(u), a^{i} \cdot e_{i}\right\rangle=\langle F(u), u\rangle
$$

and 


$$
\begin{aligned}
\|u\|_{1, p, \omega}^{p} & =\left\|\sum_{1 \leq i \leq d} a^{i} \cdot e_{i}\right\|_{1, p, \omega}^{p} \leq\left(\sum_{1 \leq i \leq d}\left|a^{i}\right| \cdot\left\|e_{i}\right\|_{1, p, \omega}\right)^{p} \\
& \leq \max _{1 \leq i \leq d}\left(\left\|e_{i}\right\|_{1, p, \omega}^{p}\right) \cdot\left(\sum_{1 \leq i \leq d}\left|a^{i}\right|\right)^{p} \\
& \leq c \cdot\|a\|_{\mathbb{R}^{p}},
\end{aligned}
$$

which implies that $\|a\|_{\mathbb{R}^{p}}$ tends to infinity if $\|u\|_{1, p, \omega}$ tends to infinity.

Now, it suffices to prove that

$$
\langle F(u), u\rangle \rightarrow \infty \quad \text { when }\|u\|_{1, p, \omega} \rightarrow \infty .
$$

Indeed, thanks to the first coercivity condition and the Hölder inequality, we obtain

$$
\begin{aligned}
I & =\int_{\Omega} \sigma(x, u, D u): D u \mathrm{~d} x \\
& \geq-\left\|\lambda_{2}\right\|_{1}-\int_{\Omega} \lambda_{3} \omega_{0 j}^{\alpha / p}\left|u_{j}\right|^{\alpha} \mathrm{d} x+c_{2} \sum_{1 \leq i, j \leq n, m} \int_{\Omega}\left|D_{i j} u\right|^{p} \omega_{i j} \mathrm{~d} x
\end{aligned}
$$

By the Hölder inequality, we have

$$
\begin{aligned}
\int_{\Omega} \lambda_{3}\left|u_{j}\right|^{\alpha} \omega_{0 j}^{\alpha / p} \mathrm{~d} x & \leq\left\|\lambda_{3}\right\|_{(p / \alpha)^{\prime}}\left(\int_{\Omega} \omega_{0 j}\left|u_{j}\right|^{(p / \alpha) \cdot \alpha}\right)^{\alpha / p} \\
& \leq c^{\prime}\left\|\lambda_{3}\right\|_{(p / \alpha)^{\prime}}\left\|u_{j}\right\|_{1, p, \omega_{0 j}} .
\end{aligned}
$$

where $c^{\prime}$ is a constant positive. For $\|u\|_{1, p, \omega}$ large enough, we can write

$$
\begin{aligned}
|I| & \geq-\left\|\lambda_{2}\right\|_{1}-c^{\prime}\left\|\lambda_{3}\right\|_{(p / \alpha)^{\prime}} \cdot\left\|u_{j}\right\|_{1, p, \omega_{0 j}}^{\alpha}+c_{2} \cdot \sum_{1 \leq i, j \leq n, m}\left\|D u_{j}\right\|_{1, p, \omega_{i j}}^{p} \\
& \geq-\left\|\lambda_{2}\right\|_{1}-c^{\prime}\left\|\lambda_{3}\right\|_{(p / \alpha)^{\prime}} \cdot\|u\|_{1, p, \omega}^{\alpha}+c_{2} c^{\prime} \cdot\|u\|_{1, p, \omega}^{p} \cdot
\end{aligned}
$$

And since

$$
\left|I^{\prime}\right|=|\langle v, u\rangle| \leq\|v\|_{-1, p^{\prime}, \omega^{*}} \cdot\|u\|_{1, p, \omega}
$$

Finally, it follows from the growth condition $\left(F_{1}\right)^{*}$ and $G_{1}$ that:

$$
\begin{aligned}
&\left|I^{\prime \prime}\right|=\left|\int_{\Omega} f(x, u, D u) \cdot u \mathrm{~d} x\right| \\
&\left.\leq \mid\left\|b_{1}\right\|_{p^{\prime}}+c_{1}^{\prime} \cdot\|D u\|_{1, p, \omega}+c_{2}^{\prime}\|D u\|_{1, p, \omega}\right) \cdot\|u\|_{1, p, \omega} \\
& \leq c_{3} \cdot\|u\|_{1, p, \omega} \\
&\left|I^{\prime \prime \prime}\right|=\left|\int_{\Omega} g(x, u) \cdot D u \mathrm{~d} x\right| \leq\left(\left\|b_{2}\right\|_{p^{\prime}}+\|u\|_{q, \gamma}^{\frac{q}{p^{\prime}}}\right) \cdot\|D u\|_{1, p, \omega} \leq c_{4} \cdot\|u\|_{1, p, \omega} ;
\end{aligned}
$$

with $c_{4}$ is a constant. With; $0<\alpha<p$ and $p>1$, we get:

$$
\begin{aligned}
I-I^{\prime}-I^{\prime \prime} \geq & c_{2} \cdot c^{\prime} \cdot\|u\|_{1, p, \omega}^{p}-\|v\|_{-1, p^{\prime}, \omega^{*}} \cdot\|u\|_{1, p, \omega}-c^{\prime}\left\|\lambda_{3}\right\|_{(p / \alpha)^{\prime}} \cdot\|u\|_{1, p, \omega}^{\alpha} \\
& -\left\|\lambda_{2}\right\|_{1}-c_{3} \cdot\|u\|_{1, p, \omega}
\end{aligned}
$$

Consequently, by using (3.1), we deduce

$$
I-I^{\prime}-I^{\prime \prime} \rightarrow \infty \text { as }\|u\|_{1, p, \omega} \rightarrow \infty .
$$

and 


$$
\begin{gathered}
I^{\prime \prime \prime} \rightarrow \infty \text { as }\|u\|_{1, p, \omega} \rightarrow \infty . \\
\langle F(u), u\rangle \rightarrow \infty \text { as }\|u\|_{1, p, \omega} \rightarrow \infty .
\end{gathered}
$$

Remark 3.2 The properties of $G$ allow us to construct our Galerkin approximations.

Corollary 3.1 For all $k \in I N$, there exists $\left(u_{k}\right) \subset V_{k}$ such that $\left\langle F\left(u_{k}\right), \varphi\right\rangle=0$, for all $\varphi \in V_{k}$.

Proof By the proposition 3.1, there exists $R>0$, such that for all $a \in \partial B_{R}(0) \subset \mathbb{R}^{d}$, we have $G(a) \cdot a>0$. And the usual topological argument see [Zei 86 proposition 2.8] [17] implies that $G(x)=0$ has a solution $x \in B_{R}(0)$. So, for all $k \in I n$, there exists $\left(u_{k}\right) \subset V_{k}$, such that

$$
\left\langle F\left(x^{j} e_{j}\right), e_{j}\right\rangle=0 \text { for all } 1 \leq j \leq d \text {, with } d=\operatorname{dim} V_{k}
$$

Taking $u_{k}=\left(x_{k}^{i} e_{i}\right), e_{i} \in V_{k}$, so we obtain:

$$
\left\langle F\left(u_{k}\right), \varphi\right\rangle=0 \text {, for all } \varphi \in V_{k} .
$$

Proposition 3.2 The Galerkin approximations sequence constructed in corollary (3.1) is uniformly bounded in $W_{0}^{1, p}\left(\Omega, \omega, \mathbb{R}^{m}\right)$; i.e.,

There exists a constant $R>0$, such that $\left\|u_{k}\right\|_{1, p, \omega} \leq R$, for all $k \in \mathbb{I N}$.

Proof Like in the proof of proposition (3.1), we can see that

$$
\langle F(u), u\rangle \rightarrow \infty \text { as }\|u\|_{1, p, \omega} \rightarrow \infty \text {. }
$$

Then, there exists $R$ satisfying $\langle F(u), u\rangle>1$ when $\|u\|_{1, p, \omega}>R$. Now, for the sequence of Galerkin approximations $\left(u_{k}\right) \subset V_{k}$ of corollary (3.1), which satisfying $\left\langle F\left(u_{k}\right), u_{k}\right\rangle=0$, we have the uniform bound $\left\|u_{k}\right\|_{1, p, \omega} \leq R$, for all $k \in \mathbb{N}$.

Remark 3.3 There exists a subsequence $\left(u_{k}\right)$ of the sequence $\left(u_{k}\right) \subset V_{k}$, such that:

$$
u_{k} \rightarrow u \text { in } W_{0}^{1, p}\left(\Omega, \omega, \mathbb{R}^{m}\right)
$$

and

$$
u_{k} \rightarrow u \text { in measure in } L^{r}\left(\Omega, \mathbb{R}^{m}\right) \text {; }
$$

with

$$
\begin{cases}1 \leq r \prec \frac{n p s}{n(s+1)-p s} & \text { if } p s \prec n(s+1) \\ r \geq 1 & \text { if } n(s+1) \prec p s\end{cases}
$$

The gradient sequence $\left(D u_{k}\right)$ generates the young measure $\vartheta_{x}$. Since $u_{k} \rightarrow u$ in measure, then $\left(u_{k}, D u_{k}\right)$ generates the Young measure $\left(\delta_{u(x)} \otimes \vartheta_{x}\right)$, see [2]. Moreover, for almost $x$ in $\Omega$, we have,

1) $\vartheta_{x}$ is the probability measure, i.e., $\left\|\vartheta_{x}\right\|_{\text {mes }}=1$.

2) $\vartheta_{x}$ is the $W^{1, p, \omega}$ gradient homogeneous young measure.

3) $\left\langle\vartheta_{x}, i d\right\rangle=D u(x)$, see [18]. 
Proof. See [2]. (Dolzmann, N. Humgerbuhler S. Muller, Non linear elliptic system ...)

\section{Passage to the Limit}

Now, we are in a position to prove our main result under convenient hypotheses.

Let

$$
I_{k}=\left(\sigma\left(x, u_{k}, D u_{k}\right)-\sigma(x, u, D u)\right):\left(D u_{k}-D u\right) .
$$

Lemma 4.1 (Fatou lemma type) (See [2]) Let. $F: \Omega \times \mathbb{R}^{m} \times I^{m \times n} \rightarrow \mathbb{R}$ be a Carathéodory function, and $u_{k}: \Omega \rightarrow \mathbb{R}^{m}$ a measurable sequence, such that $\left(D u_{k}\right)$ generates the Young measure $\vartheta_{x}$, with $\left\|\vartheta_{x}\right\|_{\text {mes }}=1$, for a.e. $x \in \Omega$. Then:

$$
\liminf _{k \rightarrow \infty} \int_{\Omega} F\left(x, u_{k}, D u_{k}\right) \mathrm{d} x \geq \int_{\Omega} \int_{M^{m \times n}} F(x, u, \zeta) \mathrm{d} \vartheta_{x}(\zeta) \mathrm{d} x,
$$

which provided that the negative part of $F\left(x, u_{k}, D u_{k}\right)$ is equi-integrable.

Proof.

Lemma 4.2 Let $p>1$ and $u_{k}$ be a sequence which is uniformly bounded in $W_{0}^{1, p}\left(\Omega, \omega, \mathbb{R}^{m}\right)$. There exists a subsequence of $u_{k}$ (for convenience not relabeled) and a function $u \in W_{0}^{1, p}\left(\Omega, \omega, \mathbb{R}^{m}\right)$ such that $u_{k} \rightarrow u$ in $W_{0}^{1, p}\left(\Omega, \omega, \mathbb{R}^{m}\right)$

And such that $u_{k} \rightarrow u$ in measure on $\Omega$ and in $L^{r}\left(\Omega, \mathbb{R}^{m}\right)$, with:

$$
\begin{cases}1 \leq r \prec \frac{n p s}{n(s+1)-p s} & \text { if } p s \prec n(s+1) \\ r \geq 1 & \text { if } n(s+1) \prec p s\end{cases}
$$

Proof. see [10].

Lemma 4.3 The sequence $\left(I_{k}\right)$ is equi-integrable.

Proof

We have

$$
\begin{aligned}
I_{k}= & \left(\sigma\left(x, u_{k}, D u_{k}\right)-\sigma(x, u, D u)\right):\left(D u_{k}-D u\right) \\
= & {\left[\sigma\left(x, u_{k}, D u_{k}\right): D u_{k}\right]-\left[\sigma\left(x, u_{k}, D u_{k}\right): D u\right] } \\
& -\left[\sigma(x, u, D u): D u_{k}\right]+[\sigma(x, u, D u): D u] \\
= & I_{k}^{1}+I_{k}^{2}+I_{k}^{3}+I_{k}^{4}
\end{aligned}
$$

We denote $\left(I_{k}^{1}\right)^{-}=-\left[\sigma\left(x, u_{k}, D u_{k}\right): D u_{k}\right]^{-}$. Thanks to the coercivity condition $\left(H_{2}\right)$, we have

$$
\begin{aligned}
\int_{\Omega^{\prime}}\left|\left(I_{k}^{1}\right)^{-}\right| \mathrm{d} x & \leq \int_{\Omega}\left|\lambda_{2}\right|+c_{2} \sum_{1 \leq j \leq m} \omega_{0 j}^{\frac{\alpha}{p}}\left|\lambda_{3}\right| \cdot\left|u_{k j}\right|^{\alpha}+c \sum_{1 \leq i, j \leq n, m} \omega_{i j}\left|D_{i j} u_{k}\right|^{p} \mathrm{~d} x \\
& \leq\left\|\lambda_{2}\right\|_{1}+\int_{\Omega^{\prime}}\left(\sum_{1 \leq j \leq m} \omega_{0 j}^{\alpha / p}\left|u_{k j}\right|^{\alpha}\right)^{p / \alpha}\left\|\lambda_{3}\right\|_{(p / \alpha)^{\prime}}+c_{2}\left\|u_{k}\right\|_{1, \omega, p}^{p}
\end{aligned}
$$

with $p / \alpha \geq 1$. Therefore,

$$
\begin{aligned}
\int_{\Omega^{\prime}}\left|\left(I_{k}^{1}\right)^{-}\right| \mathrm{d} x & \leq\left\|\lambda_{2}\right\|_{1}+\left(\sum_{1 \leq j \leq m} \omega_{0 j}\left|u_{k j}\right|^{p}\right)^{\alpha / p}\left\|\lambda_{3}\right\|_{(p / \alpha)^{\prime}}+c_{2}\left\|u_{k}\right\|_{1, \omega, p}^{p} \\
& \leq\left\|\lambda_{2}\right\|_{1}+\left\|u_{k}\right\|_{p, \bar{\omega}_{00}}^{\alpha}\left\|\lambda_{3}\right\|_{(p / \alpha)^{\prime}}+c_{2}\left\|u_{k}\right\|_{1, \omega, p}^{p} \\
& <\infty,
\end{aligned}
$$


for all $\Omega^{\prime} \subset \Omega$.

$$
\text { Similarly for }\left|\left(I_{k}^{4}\right)^{-}\right| \text {. }
$$

Now, by using the growth condition $\left(H_{2}\right)$ and the Hardy inequality $\left(H_{0}\right)$, we have

$$
\begin{aligned}
& \int_{\Omega^{\prime}}\left|\left(I_{k}^{2}\right)^{-}\right| \mathrm{d} x=\int_{\Omega^{\prime}}\left|\sigma\left(x, u_{k}, D u_{k}\right): D u_{k}\right| \mathrm{d} x \\
& \leq \beta \int_{\Omega^{\prime}} \omega_{r s}^{1 / p}\left(\lambda_{1}+c_{1} \sum_{1 \leq j \leq m} \gamma_{j}^{1 / p^{\prime}} \cdot\left|u_{k j}\right|^{q / p^{\prime}}+c_{2} \sum_{1 \leq i, j \leq n, m} \omega_{i j}^{1 / p^{\prime}}\left|D_{i j} u_{k}\right|^{p-1}\right) D_{r s} u_{k} \mathrm{~d} x .
\end{aligned}
$$

Thus, by the Hölder inequality, we obtain

$$
\begin{aligned}
\int_{\Omega^{\prime}}\left|\left(I_{k}^{2}\right)^{-}\right| \mathrm{d} x \leq & \beta\left[\left\|\lambda_{1}\right\|_{p^{\prime}}\left(\int_{\Omega^{\prime}}\left|D_{r s} u_{k}\right|^{p} \omega_{r s} \mathrm{~d} x\right)^{1 / p}\right. \\
& +c_{1}\left(\int_{\Omega^{\prime}}\left|D_{r s} u_{k}\right|^{p} \omega_{r s} \mathrm{~d} x\right)^{1 / p}\left(\int_{\Omega^{\prime}}\left(\sum_{1 \leq j \leq m} \gamma_{j}^{1 / p^{\prime}}\left|u_{k j}\right|^{q / p^{\prime}}\right)^{p^{\prime}} d x\right)^{1 / p^{\prime}} \\
& \left.+c_{1}\left(\sum_{1 \leq j \leq m} \int_{\Omega^{\prime}}\left(\left|D_{i j} u_{k}(x)\right|^{p^{\prime}(p-1)} \omega_{i j} \mathrm{~d} x\right)^{1 / p^{\prime}}\right)\left(\int_{\Omega^{\prime}}\left|D_{r s} u_{k}\right|^{p} \omega_{r s} \mathrm{~d} x\right)^{1 / p}\right] .
\end{aligned}
$$

So, by combining (4.5) and (4.6), we deduce that

$$
\int_{\Omega^{\prime}}\left|\sigma\left(x, u_{k}, D u_{k}\right): D u_{k}\right| \mathrm{d} x \leq c^{\prime} \beta\left(\left\|\lambda_{1}\right\|_{p^{\prime}}\left\|u_{k}\right\|_{1, p, \omega}+\left\|u_{k}\right\|_{1, p, \omega}\right)<\infty .
$$

Similarly to $\left|\left(I_{k}^{2}\right)^{-}\right|$, we obtain $\left|\left(I_{k}^{3}\right)^{-}\right|$. Finally: $I_{k}$ is equi-integrable.

We choose a sequence $\varphi_{k}$ such that $\varphi_{k}$ belongs to the same space $V_{k}$ and $\varphi_{k} \rightarrow \varphi$ in $W_{0}^{1, p}\left(\Omega, \omega, R^{m}\right)$, this allows us in particular, to use $u_{k}-\varphi_{k}$ as a test function in (3.1). We have:

$$
\begin{aligned}
& \int_{\Omega}\left|\sigma\left(x, u_{k}, D u_{k}\right):\left(D u_{k}-D \varphi_{k}\right)\right| \mathrm{d} x \\
& =\left\langle v, u_{k}-\varphi_{k}\right\rangle+\int_{\Omega} f\left(x, u_{k}, D u_{k}\right) \cdot\left(u_{k}-\varphi_{k}\right) \mathrm{d} x \\
& \quad-\int_{\Omega} g\left(x, u_{k}\right):\left(D u_{k}-D \varphi_{k}\right) \mathrm{d} x .
\end{aligned}
$$

The first term on the right in 4.8 converge to zero since $\left(u_{k}-\varphi_{k}\right) \rightarrow 0$ in $W_{0}^{1, p}\left(\Omega, \omega, \mathbb{R}^{m}\right)$. By the choice of $\varphi_{k}$, the sequence $\varphi_{k}$ uniformly bounded in $W_{0}^{1, p}\left(\Omega, \omega, \mathbb{R}^{m}\right)$, and lemma (4.2). Next, for the second term:

$I I_{k}=\int_{\Omega} f\left(x, u_{k}, D u_{k}\right) \cdot\left(u_{k}-\varphi_{k}\right) \mathrm{d} x$ in 4.8 it follows from the growth condition $F_{1}^{*}$ and the Hölder inequality that:

$$
\begin{aligned}
\left|I I_{k}\right| & \leq\left(\left\|b_{1}\right\|_{p^{\prime}}+c_{1}^{\prime} \cdot\left\|D\left(u_{k}-\varphi_{k}\right)\right\|_{1, p, \omega}+c_{2}^{\prime} \cdot\left\|D\left(u_{k}-\varphi_{k}\right)\right\| \frac{p}{p^{\prime}}\right) \cdot\left\|u_{k}-\varphi_{k}\right\|_{1, p, \omega} \\
& \leq\left(\left\|b_{1}\right\|_{p^{\prime}}+c \cdot\left\|D\left(u_{k}-\varphi_{k}\right)\right\|_{1, p, \omega}\right) \cdot\left\|u_{k}-\varphi_{k}\right\|_{1, p, \omega}
\end{aligned}
$$

By the equivalence of the norm in $W_{0}^{1, p}\left(\Omega, \omega, \mathbb{R}^{m}\right)$ and the sequence $u_{k}$ is uniformly bounded in $W_{0}^{1, p}\left(\Omega, \omega, \mathbb{R}^{m}\right),\left\|u_{k}\right\|_{1, p, \omega}$ is bounded.

Moreover, by the construction of $\varphi_{k}$, and lemma (4.2) we have:

$$
\begin{gathered}
\left\|u_{k}-\varphi_{k}\right\|_{1, p, \omega} \leq\left\|u_{k}-u\right\|_{1, p, \omega}+\left\|u-\varphi_{k}\right\|_{1, p, \omega} \\
\left(\left\|u_{k}-u\right\|_{1, p, \omega}+\left\|u-\varphi_{k}\right\|_{1, p, \omega}\right) \rightarrow 0
\end{gathered}
$$


We infer that the second term in 4.8 vanishes as $k \rightarrow \infty$. Finally, for the last term

$$
I I I_{k}=\int_{\Omega} g\left(x, u_{k}\right): D\left(u_{k}-\varphi_{k}\right) \mathrm{d} x
$$

in 4.8 , we note that

$$
g\left(x, u_{k}\right) \rightarrow g(x, u)
$$

Strongly in $L^{p^{\prime}}\left(\Omega, M^{m \times n}\right)$ by $\left(G_{0}\right),\left(G_{1}\right)$ and lemma (4.2).

Indeed we may assure that $u_{k} \rightarrow u$ almost everywhere.

$$
\begin{aligned}
& I I I_{k} \leq\left(\left\|b_{2}\right\|_{p^{\prime}}+\left\|u_{k}-\varphi_{k}\right\| \frac{q}{p_{q, \gamma}^{\prime}}\right) \cdot\left\|D\left(u_{k}-\varphi_{k}\right)\right\|_{1, p, \omega} \\
& \leq c^{\prime} \cdot\left(\left\|b_{2}\right\|_{p^{\prime}}+\left\|u_{k}-\varphi_{k}\right\| \frac{q}{p_{q, \gamma}^{\prime}}\right) \cdot\left\|\left(u_{k}-\varphi_{k}\right)\right\|_{1, p, \omega} \\
& \leq c^{\prime} \cdot\left(\left\|b_{2}\right\|_{p^{\prime}}+\left\|u_{k}-\varphi_{k}\right\| \|_{q, \gamma}^{\frac{q}{p^{\prime}}}\right) \cdot\left(\left\|u_{k}-u\right\|_{1, p, \omega}+\left\|\varphi_{k}-u\right\|_{1, p, \omega}\right) \\
&\left\|\varphi_{k}-u\right\|_{1, p, \omega} \rightarrow 0,\left\|u_{k}-u\right\|_{1, p, \omega} \rightarrow 0 \text { and }\left\|u_{k}-\varphi_{k}\right\| \|_{q, \gamma}^{\frac{q}{p^{\prime}}} \rightarrow 0
\end{aligned}
$$

Now, we consider $\left(I_{k}\right)^{\prime}=\sigma\left(x, u_{k}, D u_{k}\right):\left(D u_{k}-D u\right)$. We have, $I_{k}^{\prime}$ is equi-integrable because $I_{k}$ it is. So, we define

$$
\begin{aligned}
X & =\liminf \int_{\Omega} I_{k} \mathrm{~d} x=\liminf \int_{\Omega}\left(I_{k}\right)^{\prime} \mathrm{d} x \\
& \geq \int_{\Omega} \int_{\mathbb{M}^{m \times n}}(\sigma(x, u, \lambda):(\lambda-D u)) \mathrm{d} \vartheta_{x}(\lambda)
\end{aligned}
$$

So to prove (??), it suffices to prove that:

$$
X \leq 0 .
$$

Let $\varepsilon>0$, so there exists $k_{0} \in I N$ such that, for all $k>k_{0}$, we have $\operatorname{dist}\left(u, V_{k}\right)<\varepsilon$ since: $\liminf _{\varphi_{k} \in V_{k}}\left\|u-\varphi_{k}\right\|_{1, p, \omega}<\varepsilon,\left(u_{k} \rightarrow u\right)$

Or in an equivalent manner $\operatorname{dist}\left(u_{k}-u, V_{k}\right)<\varepsilon, \forall k>k_{0}$ then for all $v_{k} \in V_{k}$, we have

$$
\begin{aligned}
& X=\liminf _{k \rightarrow \infty} \int_{\Omega}\left(\sigma\left(x, u_{k}, D u_{k}\right):\left(D u_{k}-D u\right)\right) \mathrm{d} x \\
& =\liminf _{k \rightarrow \infty}\left[\int_{\Omega}\left(\sigma\left(x, u_{k}, D u_{k}\right): D\left(u_{k}-u-\varphi_{k}\right)\right) \mathrm{d} x+\int_{\Omega}\left(\sigma\left(x, u_{k}, D u_{k}\right): D\left(\varphi_{k}\right)\right)\right]
\end{aligned}
$$

Combining $\left(H_{2}\right)$ and $(0.1)$, we get

$$
\begin{aligned}
X \leq & \lim \inf _{k \rightarrow \infty} \int_{\Omega} \beta \omega_{r s}^{1 / p}\left[\lambda_{1}+c_{1} \sum_{1 \leq j \leq m} \gamma_{j}^{1 / p^{\prime}}\left|u_{k_{j}}\right|^{q / p^{\prime}}+c_{1} \sum_{1 \leq i, j \leq n, m} \omega_{i j}^{1 / p^{\prime}}\left|D_{i j} u_{k}\right|^{p-1}\right] \\
& \times\left|D_{r s}\left(u_{k}-u-\varphi_{k}\right)\right| \mathrm{d} x+\left\langle v, \varphi_{k}\right\rangle .
\end{aligned}
$$

For all $\varepsilon>0$, we choose $\varphi_{k} \in V_{k}$ such that

$$
\left\|u_{k}-u-\varphi_{k}\right\|_{1, p, \omega} \leq 2 \varepsilon
$$

For all $k \geq k_{0}$, which implies that

$$
\left|\left\langle v, \varphi_{k}\right\rangle\right| \leq\left|\left\langle v, \varphi_{k}+\left(u-u_{k}\right)\right\rangle\right|+\left|\left\langle v, u_{k}-u\right\rangle\right| \leq 2 \varepsilon\|v\|_{-1, p^{\prime}, \omega^{*}}+o(k)
$$

Hence $\lim _{k \rightarrow \infty}\left\langle v, u_{k}-u\right\rangle=0$. According to Hölder and Hardy inequalities, 
and by (4.1) we deduce that

$$
\begin{aligned}
X \leq & \liminf _{k \rightarrow \infty} c \beta\left(\left\|\lambda_{1}\right\|_{p^{\prime}}\left(\int_{\Omega}\left|D_{r s}\left(u_{k}-u-\varphi_{k}\right)\right|^{p} \cdot \omega_{r s} \mathrm{~d} x\right)^{1 / p}\right. \\
& +c_{1}\left(\int_{\Omega}\left|u_{k}\right|^{q} \cdot \gamma\right)^{1 / p^{\prime}} \cdot\left(\int_{\Omega}\left|D_{r s}\left(u_{k}-u-\varphi_{k}\right)\right|^{p} \omega_{r s} \mathrm{~d} x\right)^{1 / p} \\
& \left.+c_{1}\left(\sum \int_{\Omega} \omega_{i j}\left|D_{i j} u\right|^{p^{\prime}(p-1)}\right)^{1 / p^{\prime}} \cdot\left(\int_{\Omega} \omega_{r s}\left|D_{r s}\left(u_{k}-u-\varphi_{k}\right)\right|^{p}\right)^{1 / p}\right)+\left|\left\langle v, \varphi_{k}\right\rangle\right| \\
\leq & \liminf _{k \rightarrow \infty} c\left(\left\|\lambda_{1}\right\|_{p^{\prime}} \cdot\left\|u_{k}-u-\varphi_{k}\right\|_{1, p, \omega}\right)+\left\|u_{k}\right\|_{1, p, \omega}^{q}\left\|u_{k}-u-\varphi_{k}\right\|_{1, p, \omega} \\
& +2 \varepsilon\|v\|_{-1, p^{\prime}, \omega^{*}}+o(k)
\end{aligned}
$$

Therefore,

$$
X \leq 2 \varepsilon c \beta\left(\left\|\lambda_{1}\right\|_{p^{\prime}}+\|u\|_{1, p, \omega}^{q}+\|v\|_{-1, p^{\prime}, \omega^{*}}\right) .
$$

which proves that $X \leq 0$, and finally

$$
\int_{\Omega} \int_{\mathbb{M}^{m \times n}} \sigma(x, u, \lambda): \lambda \mathrm{d} \vartheta_{x} \mathrm{~d} x \leq \int_{\Omega} \int_{\mathbb{M}^{m \times n}} \sigma(x, u, \lambda): \operatorname{Dud} \vartheta_{x}(\lambda) \mathrm{d} x .
$$

\section{Proof of theorem:}

For arbitrary $\varphi$ in $W_{0}^{1, p}\left(\Omega, \omega, \mathbb{R}^{m}\right)$. It follows from the continuity condition $\left(F_{0}^{*}\right)$ and $\left(G_{0}\right)$ that

$$
f\left(x, u_{k}, D u_{k}\right) \cdot \varphi(x) \rightarrow f(x, u, D u) \cdot \varphi(x)
$$

and

$$
g\left(x, u_{k}\right): D \varphi(x) \rightarrow g(x, u): D \varphi(x)
$$

almost everywhere. Since, by the growth conditions $\left(F_{1}^{*}\right),\left(G_{1}\right)$ and the uniform bound of $u_{k}, f\left(x, u_{k}, D u_{k}\right) \cdot \varphi(x)$ and $g\left(x, u_{k}\right): D \varphi(x)$ are equi-integrable, it follows that the Vitali's theorem. This implies that:

$$
\lim _{k \rightarrow \infty} \int_{\Omega} f\left(x, u_{k}, D u_{k}\right) \cdot \varphi(x) \mathrm{d} x=\int_{\Omega} f(x, u, D u) \cdot \varphi(x) \mathrm{d} x
$$

for all $\varphi \in \bigcup_{k=1}^{\infty} V_{k}$ and

$$
\lim _{k \rightarrow \infty} \int_{\Omega} g\left(x, u_{k}\right): D \varphi(x) \mathrm{d} x=\int_{\Omega} g(x, u): D \varphi(x) \mathrm{d} x
$$

for all $\varphi \in \bigcup_{k=1}^{\infty} V_{k}$ We will start with the easiest case

(d): $F \mapsto \sigma(x, u, F)$ is strict p-quasi-monotone.

Indeed, we assume that $\vartheta_{x}$ is not a Dirac mass on the set $M$ with $x \in M$ of positive Lebesgue measure $|M|>0$. Moreover, by the strict p-quasi-monotonicity of $\sigma(x, u, \cdot)$ and $\vartheta_{x}$ is an homogeneous $W^{1, p}$ gradient young measure for a.e. $x \in M$. So, for a.e. $x \in M$, with $\bar{\lambda}=\left\langle\vartheta_{x}, I d\right\rangle=a p D u(x)$, with $a p D u(x)$ is the differentiable approximation in $x$. We get

$$
\begin{aligned}
& \int_{\mathbb{M}^{m \times n}} \sigma(x, u, \lambda):(\lambda-D u) \mathrm{d} \vartheta_{x}(\lambda) \\
& >\int_{\mathbb{M}^{m \times n}} \sigma(x, u, D u):(\lambda-D u) \mathrm{d} \vartheta_{x}(\lambda) \\
& >\sigma(x, u, D u): \int_{\mathbb{M}^{m \times n}} \lambda \mathrm{d} \vartheta_{x}(\lambda)-\sigma(x, u, D u): D u \int_{\mathbb{M}^{m \times n}} \mathrm{~d} \vartheta_{x}(\lambda) \\
& >(\sigma(x, u, D u): D u-\sigma(x, u, D u): D u)=0 \\
& >0
\end{aligned}
$$


On the other hand (4.9), integrating over $\Omega$, and using the div-cul inequality we have:

$$
\begin{aligned}
& \int_{\Omega} \int_{\mathbb{M}^{m \times n}} \sigma(x, u, \lambda): \lambda \mathrm{d} \vartheta_{x}(\lambda) \mathrm{d} x \\
& >\int_{\Omega} \int_{\mathbb{M}^{m \times n}} \sigma(x, u, \lambda): \operatorname{Dud} \vartheta_{x}(\lambda) \mathrm{d} x \\
& \geq \int_{\Omega} \int_{M^{m \times n}} \sigma(x, u, \lambda): \lambda \mathrm{d} \vartheta_{x}(\lambda) \mathrm{d} x .
\end{aligned}
$$

Which is a contradiction with (3.8). Thus $\vartheta_{x}=\delta_{\bar{\lambda}}=\delta_{D u(x)}$ for a.e. $x \in \Omega$. Therefore, $D u_{k} \rightarrow D u$ in measure when $k$ tends to infinity. Then, we get $\sigma\left(x, u_{k}, D u_{k}\right) \rightarrow \sigma(x, u, D u)$ for all $x \in \Omega$. In the other hand, for all $\varphi \in \bigcup \vartheta_{k} ; \sigma\left(x, u_{k}, D u_{k}\right): D \varphi \rightarrow \sigma(x, u, D u): D \varphi$ a.e. $x \in \Omega$. Moveover, for all $\Omega^{N} \subset \Omega$ measurable, it is easy to see that:

$$
\int_{\Omega^{\prime}} \sigma\left(x, u_{k}, D u_{k}\right): D \varphi \mathrm{d} x \leq c \beta\left(\left\|\lambda_{1}\right\|_{p^{\prime}}+\left\|u_{k}\right\|_{1, p, \omega}^{q / p^{\prime}}+\left\|u_{k}\right\|_{1, p, \omega}^{p / p^{\prime}}\right)\|u\|_{1, p, \omega}<\infty,
$$

because $\left\|u_{k}\right\|_{1, p, \omega} \leq R$. And thanks to Vitali's theorem, we obtain:

$$
\langle F(u), \varphi\rangle=0 \text {, for all } \varphi \in \bigcup_{k \in \mathbb{N}} \vartheta_{k} \text {. }
$$

which proves the theorem in this case.

Remark 4.1 Before treating the cases (a), (b) and (c) of $\left(H_{3}\right)$, we note that

$$
\int_{\Omega} \int_{\mathbb{M}^{m \times n}}(\sigma(x, u, \lambda)-\sigma(x, u, D u)):(\lambda-D u) \mathrm{d} \vartheta_{x}(\lambda) \mathrm{d} x \leq 0
$$

Since

$$
\int_{\Omega} \int_{M^{m \times n}} \sigma(x, u, \lambda):(\lambda-D u) \mathrm{d} \vartheta_{x}(\lambda) \mathrm{d} x=0,
$$

thanks to the div-Curl inequality in (4.9). On the other hand, the integrand in (4.12) is non negative, by the monotonicity of $\sigma$. Consequently, the integrating should be null, a.e., with respect to the product measure $\mathrm{d} \vartheta_{x} \otimes \mathrm{d} x$, which mean

$$
(\sigma(x, u, \lambda)-\sigma(x, u, D u)):(\lambda-D u)=0 \text { in } \operatorname{spt} \vartheta_{x} .
$$

Thus,

$$
\operatorname{spt} \vartheta_{x} \subset\left\{\lambda \in I M^{m \times n} /(\sigma(x, u, \lambda)-\sigma(x, u, D u)):(\lambda-D u)=0\right\} .
$$

Case c: We prove that, the map $F \mapsto \sigma(x, u, F)$ is strictly monotone, for all $x \in \Omega$ and for all $u \in \mathbb{R}^{m}$.

Sine $\sigma$ is strict monotone, and according to (4.14),

$$
\operatorname{spt} \vartheta_{x}=\{D u\} \text {, i.e, } \vartheta_{x}=\delta_{D u}, \text { a.e. in } \Omega,
$$

which implies that, $D u_{k} \rightarrow D u$ in measure. For the rest of our prove is similarly to case $\mathrm{d}$.

Case b: We start by showing that for almost all $x \in \Omega$, the support of $\vartheta_{x}$ is contained in the set where $W$ agrees with the supporting hyper-plane.

$$
L=\{(\lambda, W(x, u, \bar{\lambda})+\sigma(x, u, \bar{\lambda}):(\lambda-\bar{\lambda}))\} \text { with } \bar{\lambda}=D u(x) .
$$

So, it suffices to prove that 
$\operatorname{spt} \vartheta_{x} \subset K_{x}=\left\{\lambda \in I M^{m \times n} / W(x, u, \lambda)=W(x, u, \bar{\lambda})+\sigma(x, u, \bar{\lambda}):(\lambda-\bar{\lambda})\right\}$

If $\lambda \in \operatorname{spt} \vartheta_{x}$, thanks to (4.14), we have

$$
(1-t) \cdot(\sigma(x, u, D u)-\sigma(x, u, \lambda)):(D u-\lambda)=0 \text {, for all } t \in[0,1] \text {. }
$$

On the other hand, since $\sigma$ is monotone, for all $t \in[0,1]$ we have:

$$
(1-t) \cdot(\sigma(x, u, D u+t \cdot(\lambda-D u))-\sigma(x, u, \lambda)):(D u-\lambda) \geq 0 \text {. }
$$

By subtracting (4.16) from (4.17), we get

$$
(1-t)[\sigma(x, u, \bar{\lambda}+t(\lambda-\bar{\lambda}))-\sigma(x, u, \bar{\lambda})]:(\bar{\lambda}-\lambda) \geq 0,
$$

for all $t \in[0,1]$. Doing the same by the monotonicity in (4.18), we obtain

$$
(1-t)[\sigma(x, u, \bar{\lambda}+t(\lambda-\bar{\lambda}))-\sigma(x, u, \bar{\lambda})]:(\bar{\lambda}-\lambda) \leq 0 .
$$

Combining (4.18) and (4.19), we conclude that

$$
(1-t)[\sigma(x, u, \bar{\lambda}+t(\lambda-\bar{\lambda}))-\sigma(x, u, \bar{\lambda})]:(\bar{\lambda}-\lambda)=0,
$$

for all $t \in[0,1]$, and for all $\lambda \in \operatorname{spt} \vartheta_{x}$.

Now, it follows from (4.19) that

$$
\begin{aligned}
W(x, u, \lambda) & =W(x, u, \bar{\lambda})+(W(x, u, \lambda)-W(x, u, \bar{\lambda})) \\
& =W(x, u, \bar{\lambda})+\int_{0}^{1}[\sigma(x, u, \bar{\lambda})+t(\lambda-\bar{\lambda})]:(\lambda-\bar{\lambda}) \mathrm{d} t \\
& =W(x, u, \bar{\lambda})+\sigma(x, u, \bar{\lambda}):(\lambda-\bar{\lambda})
\end{aligned}
$$

Witch prove (4.15).

Now, by the coercivity of $W$, we get

$$
W(x, u, \lambda) \geq W(x, u, \bar{\lambda})+\sigma(x, u, \bar{\lambda}):(\lambda-\bar{\lambda}),
$$

for all $\lambda \in I M^{m \times n}$. Therefore,

$L$ is a supporting hyper-plane, for all

$$
\lambda \in K_{x} .
$$

Moveover, the mapping $\lambda \mapsto W(x, u, \lambda)$ is continuously differentiable, so we obtain

$$
\sigma(x, u, \lambda)=\sigma(x, u, \bar{\lambda}) \text {, for all } \lambda \in K_{x}
$$

Thus,

$$
\bar{\sigma}(x)=\int_{\mathbb{M}^{m \times n}} \sigma(x, u, \lambda) \mathrm{d} \vartheta_{x}(\lambda)=\sigma(x, u, \bar{\lambda}) .
$$

Now, we consider the Carathéodory function

$$
g_{v}(x, u, \rho)=|(\sigma(x, u, \rho)-\bar{\sigma}(x)): D \varphi|,
$$

and lets $g_{k}(x)=g_{\varphi}\left(x, u_{k}, D u_{k}\right)$ is equi-integrable. Thus, thanks to BALL's theorem, see [6] $g_{k} \rightarrow g$ weakly in $L^{1}(\Omega)$, and the weakly limit of $g$ is given by

$$
\begin{aligned}
\bar{g}_{\varphi}(x) & =\iint_{\mathbb{R}^{m} \times \mathbb{M}^{m \times n}}|\sigma(x, \eta, \lambda)-\bar{\sigma}(x)| \mathrm{d} \delta_{u(x)}(\eta) \otimes \mathrm{d} \vartheta_{x}(\lambda) \\
& =\int_{s p t \vartheta_{x}}|\sigma(x, u(x), \lambda)-\bar{\sigma}(x)| \mathrm{d} \vartheta_{x}(\lambda) \\
& =0 .
\end{aligned}
$$


According to (4.22) and (4.23), and since $g_{k} \geq 0$, it follow that $g_{k} \rightarrow 0$ strongly in $L^{1}(\Omega)$ by Fatou lemma, which gives

$$
\lim _{k \rightarrow \infty} \int_{\Omega} \sigma\left(x, u_{k}, D u_{k}\right): D \varphi \mathrm{d} x=\int_{\Omega} \sigma(x, u, D u): D \varphi \mathrm{d} x .
$$

Thus

$$
\langle F(u), \varphi\rangle=0, \forall \varphi \in \bigcup_{k \in \mathbb{N}} V_{k} .
$$

This completes the proof of the case (b).

Case (a): In this case, on $\operatorname{spt} \vartheta_{x}$, we affirm that,

$$
\sigma(x, u, \lambda): M=\sigma(x, u, D u): M+\left(\nabla_{F} \sigma(x, u, D u): M\right):(D u-\lambda),
$$

for all $M \in I M^{m \times n}$, where $\nabla_{F}$ is the derivative with respect to the third variable of $\sigma$ and $\bar{\lambda}=D u(x)$.

Thanks to the monotonicity of $\sigma$, we have

$$
(\sigma(x, u, \lambda)-\sigma(x, u, D u+t M)):(\lambda-D u-t M) \geq 0 \text {, for all } t \in \mathbb{R} \text {. }
$$

By invoking (4.19), we obtain

$$
\begin{aligned}
& -\sigma(x, u, \lambda):(t M) \\
& \geq-\sigma(x, u, D u):(\lambda-D u)+\sigma(x, u, D u+t M):(\lambda-D u-t \cdot M) .
\end{aligned}
$$

On the other hand, $F \mapsto \sigma(x, u, F)$ is a $C^{1}$ function, so

$$
\sigma(x, u, D u+t M)=\sigma(x, u, D u)+\nabla_{F}(x, u, D u) \cdot(t M)+o(t) .
$$

Thus

$$
\begin{aligned}
& -\sigma(x, u, \lambda):(t \cdot M) \\
& \geq-\sigma(x, u, D u):(t M)+\nabla_{F} \sigma(x, u, D u)(t \cdot M):(\lambda-D u)+o(t),
\end{aligned}
$$

which gives

$$
\begin{aligned}
& -\sigma(x, u, \lambda):(t \cdot M) \\
& \geq t\left[\nabla_{F} \sigma(x, u, D u):(M):(\lambda-D u)-\sigma(x, u, D u):(M)\right]+o(t),
\end{aligned}
$$

$t$ is arbitrary in (4.24).

Finally for all $\varphi \in \bigcup_{k \in \mathbb{N}} V_{k}$ the sequence $\sigma\left(x, u_{k}, D u_{k}\right): D \varphi$ is equi-integrable. Then, by the BALL's theorem, see [1] the weak limit is $\int_{s p t \vartheta_{x}} \sigma(x, u, \lambda): D \varphi \mathrm{d} \vartheta_{x}(\lambda)$

$$
\begin{aligned}
& \text { By choosing } M=D u \text { in }(4.24) \text {, we obtain } \\
& \int_{\text {spt } \vartheta_{x}}(D u-\lambda)(\sigma(x, u, \lambda): D \varphi): D \varphi \mathrm{d} \vartheta_{x}(\lambda) \\
& =\int_{s p t \vartheta_{x}} \sigma(x, u, D u): D \varphi \mathrm{d} \vartheta_{x}(\lambda)+\left(\nabla_{F} \sigma(x, u, D u): D \varphi\right)^{t} \int_{s p t \vartheta_{x}}(D u-\lambda) \mathrm{d} \vartheta_{x}(\lambda) \\
& =(\sigma(x, u, D u): D \varphi) \int_{s p t \vartheta_{x}} \mathrm{~d} \vartheta_{x}(\lambda)=\sigma(x, u, D u): D \varphi .
\end{aligned}
$$

Hence:

$$
\sigma\left(x, u_{k}, D u_{k}\right): D \varphi \rightarrow \sigma(x, u, D u): D \varphi \text { strongly }
$$

This proves that

$$
\langle F(u), \varphi\rangle=0 \text { for all } \varphi \in \bigcup V_{k} \text {. }
$$


And since $\bigcup V_{k}$ is dense in $W_{0}^{1, P}\left(\Omega, \omega, \mathbb{R}^{m}\right)$, so $u$ is a weak solution of $(Q E S)_{f, g}^{*}$, as desired.

Remark 4.2 In case (b) $\sigma\left(x, u_{k}, D u_{k}\right): D \varphi \rightarrow \sigma(x, u, D u): D \varphi$ strongly, but in the case (c) and (d) $D u_{k} \rightarrow D u$ in measure.

Exemple 4.1 We shall suppose that the weight functions satisfy: $w_{i_{0} j}=0, j=1,2, \cdots, m$ for some $i_{0} \in I^{c}$; and $\omega_{i j}(x)=w(x) ; x \in \Omega$, with $I^{c} \bigcup I=\{0 ; 1 ; 2 ; \cdots ; n\}$, for all $i \in I \sqcup I^{c}, \quad j=1,2, \cdots, m$, and $i \neq i_{0}$ with $w(x)>0$ a.e in $\Omega$ then, we can consider the Hardy inequality in the form:

$$
\left(\sum_{j=1}^{m} \int_{\Omega}\left|u_{j}(x)\right|^{q} \gamma_{j}(x) \mathrm{d} x\right)^{\frac{1}{q}} \leq c\left(\sum_{1 \leq i \leq N, 1 \leq j \leq m} \int_{\Omega}\left|D_{i j} u\right|^{p} \omega_{i j}\right)^{\frac{1}{p}},
$$

for every $u \in W_{0}^{1, p}\left(\Omega, \omega, \mathbb{R}^{m}\right)$ with a constant $c>0$ independent of $u$ and for some $q>p^{\prime}$. Let us consider the Carathéodory functions. $(\star)$

$$
\begin{gathered}
\sigma_{i j}\left(x, \eta, \xi_{I}\right)=\omega(x)\left|\xi_{i j}\right|^{p-1} \operatorname{sng}\left(\xi_{i j}\right), j=1,2, \cdots, m, i \in I \\
\sigma_{i j}\left(x, \eta, \xi_{I^{c}}\right)=\omega(x)\left|\xi_{i j}\right|^{p-1} \operatorname{sng}\left(\xi_{i j}\right), j=1,2, \cdots, m, i \in I^{c}, i \neq i_{0} \\
\sigma_{i_{0} j}\left(x, \eta, \xi_{I^{c}}\right)=0, j=1,2, \cdots, m \\
f_{j}(x, \eta, \xi)=-\operatorname{sign}(\xi) \sum_{r s} \omega_{r s}^{\frac{1}{p^{p}}}|\xi|^{p-1} \omega_{0 j}^{\frac{1}{p}}
\end{gathered}
$$

The above functions defined by $(\star)$ satisfies the growth conditions $\left(H_{2}\right)$.

In particular, let use the special weight function $\omega, \gamma$ expressed in term of the distance to the boundary $\partial \Omega$ denote $d(x)=\operatorname{dist}(x ; \partial \Omega)$ and $\omega(x)=d^{\lambda}(x), \gamma_{j}(x)=d^{\mu}(x)$ the hardy inequality reads.

$$
\left(\sum_{j=1}^{m} \int_{\Omega}\left|u_{j}(x)\right|^{q} \mathrm{~d}^{\mu}(x) \mathrm{d} x\right)^{\frac{1}{q}} \leq c\left(\sum_{1 \leq i \leq N, 1 \leq j \leq m} \int_{\Omega}\left|D_{i j} u\right|^{p} \mathrm{~d}^{\lambda}(x)\right)^{\frac{1}{p}},
$$

and the corresponding $W_{0}^{1, p}\left(\Omega ; \omega ; R^{m}\right) \hookrightarrow L^{q}\left(\Omega ; \gamma ; R^{m}\right)$ is compact if:

1) For, $1<p \leq q<\infty$

$$
\lambda<p-1 ; \frac{n}{q}-\frac{n}{p}+1 \geq 0 ; \frac{\mu}{q}-\frac{\lambda}{p}+\frac{n}{q}-\frac{n}{p}+1>0
$$

2) For, $1 \leq q<p<\infty$

$$
\lambda<p-1 ; \frac{n}{q}-\frac{n}{p}+1 \geq 0 ; \frac{\mu}{q}-\frac{\lambda}{p}+\frac{1}{q}-\frac{1}{p}+1>0
$$

3) For, $q>1$

$\mu\left(q^{\prime}-1\right)<1$, by the simple modifications of the example in [11]. Moreover, the monotonicity condition are satisfied:

$$
\begin{aligned}
& \sum_{i j}\left(\sigma_{i j}\left(x, \eta, \xi_{I}\right)-\sigma_{i j}\left(x, \eta, \xi_{I}^{\prime}\right)\right)\left(\xi_{i j}-\xi_{i j}^{\prime}\right) \\
& =\omega(x) \sum_{i j}\left(\left|\xi_{i j}\right|^{p-1} \operatorname{sng}\left(\xi_{i j}\right)-\left|\xi_{i j}^{\prime}\right|^{p-1} \operatorname{sng}\left(\xi_{i j}^{\prime}\right)\right)\left(\xi_{i j}-\xi_{i j}^{\prime}\right) \geq 0
\end{aligned}
$$

for almost all $x \in \Omega$ and for all, $\xi, \xi^{\prime} \in M^{n}$. This last inequality cannot be 
strict, since for $\xi_{I^{c}} \neq \xi_{I^{c}}^{\prime}$ with $\xi_{i_{0} j} \neq \xi_{i_{0} j}^{\prime}$ for all $j=1,2, \cdots, m$. But $\xi_{i j}=\xi_{i j}^{\prime}$ for $i \in I^{c}, i \neq i_{0}, \quad j=1,2, \cdots, m$ the corresponding expression is Zero.

\section{Conflicts of Interest}

The authors declare no conflicts of interest regarding the publication of this paper.

\section{References}

[1] Ball, J. (1989) A Version of the Fundamental Theorem for Young Measures. In Partial Differential Equations and Continuum Models of Phase Transitions. Proceedings of an NSF-CNRS Joint Seminar, Nice, France, 207-215.

[2] Dolzmann, G., Hungerbuhler, N. and Muller, S. (1997) Nonlinear Elliptic Systems with Measure-Valued Right Hand Side. Mathematische Zeitschrift, 226, 545-574. https://doi.org/10.1007/PL00004354

[3] Minty, G.J. (1962) Monotone (Nonlinear) Operators in Hilbert Space. Duke Mathematical Journal, 29, 341-346. https://doi.org/10.1215/S0012-7094-62-02933-2

[4] Brezis, H. (1973) Operateurs maximaux monotones et semi-groupes de contractions dans les espaces de Hilbert. North-Holland Mathematics Studies, No. 5. Notas de Matematica (50). North-Holland Publishing Co., Amsterdam-London, American Elsevier Publishing Co. Inc., New York.

[5] Edwin, H. and Stromberg, K. (1969) Real and Abstract Analysis. Springer-Verlag, Berlin.

[6] Fonseca, I., Muller, S. and Pedregal, P. (1998) Analysis of Concentration and Oscillation Effects Generated by Gradients. SIAM Journal on Mathematical Analysis, 29, 736-756. https://doi.org/10.1137/S0036141096306534

[7] Lions, J.L. (1969) Quelques Methodes de Resolution des Problemes aux Limites non Lineaires. Dunod, Gauthier-Villars, Paris.

[8] Kristznsen, J. (1999) Lower Semicontinuity in Space of Weakly Differentiable Functions. Mathematische Annalen, 313, 653-710. https://doi.org/10.1007/s002080050277

[9] Visik, M.I. (1963) Quasi-Linear Strongly Elliptic Systems of Differential Equations of Divergence Form. Trudy Moskov. Mat. Obsc, 12, 125-184. (In Russian)

[10] Hungerbühler, N. (1999) Quasilinear Elliptic Systems in Divergence form with Weak Monotonicity. New York Journal of Mathematics, 5, 83-90.

[11] Akdim, Y. and Azroul, E. (2002) Pseudo-Monotonicity and Degenerated Elliptic Operator of Second Order. Southwest Texas State University, San Marcos.

[12] Benilan, P., Brezis, H. and Crandall, M.G (1975) A Semilinear Equation in L ${ }^{1}$. Annali della Scuola Normale Superiore di Pisa-Classe di Scienze, 2, 523-555.

[13] Fabrie, P. and Gallouet. T (2000) Modelling Wells in Porous Media Flows. Mathematical Models and Methods in Applied Sciences, 10, 673-709. https://doi.org/10.1142/S0218202500000367

[14] Kinderlehrer, D. and Pedregal, P. (1994) Gradient Young Measures Generated by Sequences in Sobolev Spaces. The Journal of Geometric Analysis, 4, Article No. 59. https://doi.org/10.1007/BF02921593

[15] Browder, F.E. (1970) Existence Theorems for Nonlinear Partial Differential Equations. Global Analysis (Berkeley, 1968). In: Proceedings of Symposia in Pure Mathematics Publication, Vol. 16, AMS, Providence, 1-60. 
https://doi.org/10.1090/pspum/016/0269962

[16] Drabek, P., Kufnen, A. and Mustonen, V. (1998) Pseudo-Monotinicity and Degenerated, a Singular Operators. Bulletin of the Australian Mathematical Society, 58, 213-221. https://doi.org/10.1017/S0004972700032184

[17] Landes, R. and Mustonen, V. (1980) On Pseudo-Monotone Operators and Nonlinear Noncoercive Variational Problems on Unbounded Domains. Mathematische Annalen, 248, 241-246. https://doi.org/10.1007/BF01420527

[18] Zeidler, E. (1986) Nonlinear Functional Analysis and Its Application. Vol. I, Springer, New York. 